\title{
O Tratamento Fisioterapêutico na Doença de Parkinson
}

\author{
Physical Therapy in Parkinson's Disease \\ Andressa Correa Vara', Renata Medeiros², Vera Lúcia Widniczck Striebel
}

\section{RESUMO}

\begin{abstract}
A Doença de Parkinson (DP) é uma doença neurológica progressiva do sistema extrapiramidal caracterizada por bradicinesia, tremor de repouso, rigidez e instabilidade postural. O objetivo desta revisão é demonstrar o benefício da prática de atividade física regular no paciente com DP, melhorando sua qualidade de vida. Método. Foi realizado uma revisão sistemática das publicaçôes indexadas em bases de dados Pubmed, Scielo, Scopus, Capes e Cochrane, no período de 1996 a 2009 nos idiomas em inglês e português. Resultados. Embora não existam trabalhos científicos com tamanho de amostra muito significativo, as pesquisas até agora existentes demonstram que as intervençôes de exercícios físicos auxiliam, e que não devem ser em curto prazo, e sim tornar-se parte do estilo de vida diário. Conclusáo. A fisioterapia, orientando a prática de atividade física regular é de extrema importância para manter, melhorar e prolongar a qualidade de vida do indivíduo, estando evidenciado que a administração de atividades físicas regulares em pacientes com DP merece consideração.
\end{abstract}

Unitermos. Doença de Parkinson, Fisioterapia Neurológica, Atividade Motora, Exercício.

Citaçáo. Vara AC, Medeiros R, Striebel VLW. O Tratamento Fisioterapêutico na Doença de Parkinson.

\begin{abstract}
Parkinson`s Disease (PD) is a progressive neurological disorder of the extrapyramidal system characterized by bradykinesia, resting tremor, rigidity and postural instability. Method. It was conducted a systematic review of publications indexed in databases such as Pubmed, Scielo, Scopus, Capes and Cochrane, in the period of 1996 to 2009 in English and Portuguese languages. Results. Although there are no scientific studies with sample size, previous studies have already demonstrated that chronic physical exercise are benefic, and should become part of daily lifestyle. Conclusion. Physical therapy, guiding the practice of physical activity is extremely important to maintain, improve and prolong quality of life of the individual, being shown that the administration of regular physical activity in PD patients deserves consideration.
\end{abstract}

Keywords. Parkinson's Disease, Neurological Physiotherapy, Motor Activity, Exercise.

Citation. Vara AC, Medeiros R, Striebel VLW. Physical Therapy in Parkinson's Disease.
Trabalho realizado no Laboratório de Fisiologia do Exercício do Centro Universitário Metodista - IPA, Porto Alegre- RS, Brasil.

1. Acadêmica do Curso de Fisioterapia do Centro Universitário MetodistaIPA, Porto Alegre-RS, Brasil.

2. Acadêmica do Curso de Fisioterapia do Centro Universitário MetodistaIPA, Porto Alegre-RS, Brasil.

3. Mestre, Professora do Centro Universitário Metodista- IPA, Porto AlegreRS, Brasil.
Endereço para correspondência: Renata Medeiros

Rua Miguel Ascoleze, No57, bairro Vila Nova CEP 91740-470, Porto Alegre-RS, Brasil.

E-mail: renatanoal@terra.com.br

Revisão

Recebido em: 18/10/10

Aceito em: 28/07/11

Conflito de interesses: não 


\section{INTRODUÇÃO}

A Doença de Parkinson (DP) é uma doença neurológica progressiva do sistema extrapiramidal caracterizada por bradicinesia, tremor de repouso, rigidez e instabilidade postural. Outros achados clínicos importantes são: distúrbio da marcha, fácies em máscara, alteração da voz, disartria, sialorréia, disfunção olfatória, hipotensão ortostática, hiperidrose, seborréia, disfunção sexual, câimbras, dores, parestesias, disfagia, incontinência urinária, micrografia, distúrbios do sono, bradifrenia, depressão e demência ${ }^{1-3}$.

O início do quadro clínico ocorre geralmente entre 50 e 70 anos de idade. Contudo, podem-se encontrar pacientes com início da doença mais precoce, antes dos 40 anos e até mesmo abaixo dos 21 anos de idade ${ }^{4}$.

Esta enfermidade degenerativa cursa com uma perda progressiva de células da substância negra do mesencéfalo. A degeneração de neurônios da zona compacta da substância negra resultará numa diminuição da produção de dopamina, com disfunção da via nigroestriatal e subsequente perda da dopamina estriatal. Quando aproximadamente $80 \%$ dos neurônios da substância negra forem perdidos, a DP torna-se evidente e os indivíduos começam a experimentar uma grande variedade de dificuldades $^{5,6}$.

Existem fases durante o período de síntese, liberação e metabolismo da dopamina dentro do sistema nervoso central, cuja intervenção medicamentosa é aumentando os níveis de dopamina, que podem influenciar as manifestaçôes clínicas da $\mathrm{DP}^{2}$.

Mesmo antes do diagnóstico de DP ter sido confirmado por um neurologista, a maioria das pessoas com a doença terá a consciência de que estão caminhando mais vagarosamente que o usual e com passos menores. Previamente ao início do uso de levodopa, neste estágio mais inicial, há uma oportunidade de acessar os níveis mais básicos, iniciais, dos prejuízos, limitações de atividades e restrição de participação antes do início das medicaçóes. A variação natural na capacidade da pessoa, portando, pode ser medida, sem os efeitos da terapêutica farmacológica. Dados de base podem ser registrados para posterior uso na avaliação da progressão da doença ${ }^{7}$.

O diagnóstico é primariamente clínico, baseado na história médica e exame físico. Com o desenvolvimen- to de novos tratamentos para a DP, tornou-se necessário criar e desenvolver escalas para avaliar a doença. Essas escalas avaliam desde a condição clínica geral, incapacidades, função motora e mental até a qualidade de vida dos pacientes. Tais instrumentos são importantes, pois permitem monitorar a progressão da doença e a eficácia de tratamentos e drogas ${ }^{8}$.

Entre os indivíduos com DP, as quedas são muito frequentes. Cerca de $65 \%$ dos que caem irão experimentar uma injúria secundária a suas quedas, $33 \%$ sofrerão uma fratura e $75 \%$ das quedas conduzirão o paciente a procurar um serviço de saúde. Queda com fratura é a causa mais comum de atendimento hospitalar para estes indivíduos. Estas quedas têm consequências devastadoras e são acompanhadas de dor, redução da mobilidade e elevados níveis de estresse. Estas quedas desenvolvem um temor, o qual determina restrição das atividades e comprometimento da qualidade de vida, predispondo a redução secundária na força muscular e na capacidade cardiovascular'.

O tratamento da DP é baseado no uso de terapia medicamentosa que influencia marcadamente a desempenho motor, contudo o tratamento com drogas náo pode abolir todos os sintomas, e fisioterapia é então recomendada. Dependendo da concentração sérica do medicamento, o paciente terá um período "on" (com efeito máximo da droga) e um período "off” (com o mínimo efeito da droga). Pacientes no período "on" estão mais capacitados a realizarem exercícios físicos, portanto o uso da medicação deve ser ajustado ao inicio de uma atividade de maior esforço ${ }^{10}$.

A abordagem cirúrgica da DP tem quase um século, mas é usada somente quando o paciente não é responsivo às medidas farmacológicas ou desenvolvem reaçôes adversas intoleráveis a medicações antiparkinsonismo. As medidas não farmacológicas compreendem uma série de hábitos e medidas de valor especial na doença, por minimizarem algumas de suas complicaçóes. Tais medidas são a educaçáo, tratamento de suporte, exercício e nutriçãó ${ }^{4}$.

A fisioterapia voltada para a DP tem como objetivo minimizar os problemas motores, ajudando o paciente a manter a independência para realizar as atividades de vida diária e melhorando sua qualidade de vida. Com o 
exercício, o aumento da mobilidade pode de fato modificar a progressão da doença e impedir contraturas, além de ajudar a retardar a demência ${ }^{4}$. Assim, o objetivo desta revisão é descrever os efeitos da fisioterapia na DP.

\section{MÉTODO}

Realizou-se no período de março a julho de 2009 uma revisão sistemática das publicações indexadas nas bases de dados Medline, Scielo, Scopus, Capes e Cochrane, no período de 1996 a 2009, nos idiomas inglês e português. Utilizou-se as seguintes palavras-chave: Doença de Parkinson, Parkinson's Disease, exercício físico, physical exercise, training, maximal oxygen. Os critérios de inclusão foram estudos que abordavam a fisiopatologia, o tratamento medicamentoso, a atividade física e a fisioterapia. Critérios de exclusão foram artigos com animais e aqueles que não apresentavam clareza quanto ao método utilizado e resultados.

\section{RESULTADOS}

Neste estudo de revisão de literatura foram encontrados 39 estudos no total. Destes, 11 artigos foram excluídos por se tratarem de temas que não abordavam interesse fisioterapêutico. Entre os 28 artigos restantes, 3 foram excluídos por praticarem exercícios em modelos animais, restando assim, 21 artigos encontrados na base de dados Pubmed, Scielo, Scopus, Capes e Cochrane. A Tabela 1 apresenta um breve resumo dos artigos usados.

Tabela 1

Resumo dos artigos encontrados

\begin{tabular}{|c|c|c|c|c|}
\hline ARTIGO & AUTOR & ANO & OBJETIVO & CONCLUSÁO \\
\hline $\begin{array}{c}\text { Locomotor Training in } \\
\text { People With Parkinson } \\
\text { Disease. } \\
\end{array}$ & Morries ME. $^{7}$ & 2006 & $\begin{array}{l}\text { É considerar o papel do } \\
\text { fisioterapeuta no treinamento } \\
\text { motor de portadores da DP. }\end{array}$ & $\begin{array}{c}\text { Parte do programa de treinamen- } \\
\text { to deve otimizar a qualidade de } \\
\text { vida e vida social. }\end{array}$ \\
\hline $\begin{array}{l}\text { Movement disorders in } \\
\text { people with Parkinson di- } \\
\text { sease: a model for physical } \\
\text { therapy. }\end{array}$ & Morries ME. ${ }^{11}$ & 2000 & $\begin{array}{l}\text { Apresentar um modelo de } \\
\text { gerenciamento da fisioterapia } \\
\text { para pessoas com DP idiopá- } \\
\text { tica baseado no conhecimento } \\
\text { da patogênese dos distúrbios } \\
\text { de movimento desta doença. }\end{array}$ & $\begin{array}{l}\text { A intervenção fisioterapêutica } \\
\text { junto ao medicamento tem o } \\
\text { potencial de reduzir os sintomas } \\
\text { da DP. Melhorando a qualidade } \\
\text { de vida destes indivíduos. }\end{array}$ \\
\hline $\begin{array}{l}\text { Therapeutic value of exer- } \\
\text { cise training in Parkinson`s } \\
\text { disease. }\end{array}$ & Reuter I. ${ }^{12}$ & 1999 & $\begin{array}{l}\text { Investigar a influência de um } \\
\text { treinamento intensivo na } \\
\text { incapacidade motora, humor e } \\
\text { bem-estar na DP. }\end{array}$ & $\begin{array}{l}\text { Os portadores de DP através da } \\
\text { realizaçáo de exercícios físicos } \\
\text { podem melhorar seu humor, } \\
\text { bem- estar e incapacidade moto- } \\
\text { ra na doença. }\end{array}$ \\
\hline $\begin{array}{c}\text { Conhecimento e percepção } \\
\text { sobre exercício físico em } \\
\text { uma populaçáo adulta } \\
\text { urbana do sul do Brasil. }\end{array}$ & Domingues MR. ${ }^{13}$ & 2004 & $\begin{array}{c}\text { Avaliar o conhecimento e a } \\
\text { percepçáo sobre exercício físi- } \\
\text { co em uma populaçáo adulta } \\
\text { urbana. }\end{array}$ & $\begin{array}{c}\text { Independente do sexo existe } \\
\text { uma forte associaçáo entre este } \\
\text { conhecimento e o nível social e a } \\
\text { escolaridade. }\end{array}$ \\
\hline $\begin{array}{l}\text { The Impact of Exercise } \\
\text { Rehabilitation and Physical } \\
\text { Activity on the Mana- } \\
\text { gement of Parkinson's } \\
\text { disease. }\end{array}$ & Johnson AM. ${ }^{14}$ & 2007 & $\begin{array}{l}\text { Estudar o uso de exercícios } \\
\text { motores na DP. }\end{array}$ & $\begin{array}{l}\text { Os portadores de DP devem } \\
\text { ser incentivados a manter uma } \\
\text { atividade física juntamente com } \\
\text { o tratamento farmacológico. }\end{array}$ \\
\hline $\begin{array}{l}\text { Benefícios do treinamento } \\
\text { resistido na reabilitação da } \\
\text { marcha e equilíbrio nos } \\
\text { portadores da Doença de } \\
\text { Parkinson. }\end{array}$ & Braga A. ${ }^{15}$ & 2003 & $\begin{array}{l}\text { Mostrar que a reabilitação da } \\
\text { marcha e equilíbrio do doente } \\
\text { de Parkinson requer vários } \\
\text { recursos e abordagens. }\end{array}$ & $\begin{array}{l}\text { Concluiu-se que exercícios } \\
\text { resistidos devem ser aplicados } \\
\text { no tratamento dos portadores } \\
\text { de DP. }\end{array}$ \\
\hline $\begin{array}{c}\text { Nonpharmacologic } \\
\text { management of Parkinson } \\
\text { disease. }\end{array}$ & Tarsy D. ${ }^{16}$ & 2008 & $\begin{array}{l}\text { Analisar o tratamento náo } \\
\text { farmacológico na DP. }\end{array}$ & $\begin{array}{l}\text { As necessidades emocionais e psi- } \\
\text { cológicas do paciente e da família } \\
\text { devem ser abordadas. }\end{array}$ \\
\hline $\begin{array}{l}\text { Multidiciplinary rehabi- } \\
\text { litation for people with } \\
\text { Parkinson`s disease: a ran- } \\
\text { domized controlled study. }\end{array}$ & Wade DT. ${ }^{17}$ & 2002 & $\begin{array}{l}\text { Determinar se um programa } \\
\text { de reabilitaçáo multidisci- } \\
\text { plinar e grupo de apoio tem } \\
\text { benefício para portadores da } \\
\text { DP ou seus cuidadores. }\end{array}$ & $\begin{array}{l}\text { Um curto período de reabilitação } \\
\text { multidisciplinar pode ampliar } \\
\text { sua mobilidade. Seguir o trata- } \\
\text { mento pode ser necessário para } \\
\text { manter algum benefício. }\end{array}$ \\
\hline
\end{tabular}




\begin{tabular}{|c|c|c|c|c|}
\hline ARTIGO & AUTOR & ANO & OBJETIVO & CONCLUSÁO \\
\hline $\begin{array}{l}\text { Delaying mobility disabili- } \\
\text { ty in people with Parkinson } \\
\text { disease using a senso- } \\
\text { rimotor agility exercise } \\
\text { program. }\end{array}$ & King LA. ${ }^{18}$ & 2009 & $\begin{array}{l}\text { Desenvolver um programa } \\
\text { de exercícios para minimizar } \\
\text { a incapacidade funcional em } \\
\text { pessoas com DP. }\end{array}$ & $\begin{array}{l}\text { Um programa de mobilidade, } \\
\text { como o apresentado neste artigo, } \\
\text { teria de ser sustentada e modi- } \\
\text { ficados ao longo do curso da } \\
\text { doença para manter o benefício } \\
\text { máximo. }\end{array}$ \\
\hline $\begin{array}{l}\text { Uma análise do compro- } \\
\text { metimento da fala em } \\
\text { portadores de doença de } \\
\text { Parkinson. }\end{array}$ & Barros ALS. ${ }^{19}$ & 2004 & $\begin{array}{l}\text { Analisar a fala, enquanto pro- } \\
\text { cesso articulatório em sujeitos } \\
\text { com DP. }\end{array}$ & $\begin{array}{c}\text { O tratamento precoce em sujei- } \\
\text { tos em estágio inicial possibilita } \\
\text { a atenuação das alteraçóes da } \\
\text { articulaçáo da fala. }\end{array}$ \\
\hline $\begin{array}{l}\text { Physiotherapy for patients } \\
\text { with Parkinson's disease. }\end{array}$ & Deane K. ${ }^{20}$ & 2001 & $\begin{array}{l}\text { Comparar a eficácia e a efeti- } \\
\text { vidade de algumas técnicas de } \\
\text { fisioterapia. }\end{array}$ & $\begin{array}{l}\text { Não foi possível comprovar qual } \\
\text { técnica da fisioterapia apresenta } \\
\text { melhor resultado para a DP. }\end{array}$ \\
\hline $\begin{array}{l}\text { The effectiveness of exercise } \\
\text { interventions for people } \\
\text { with Parkinon`s disease: } \\
\text { a systematic review and } \\
\text { meta-analysis. }\end{array}$ & Goodwin VA. $^{21}$ & 2008 & $\begin{array}{c}\text { Revisar sistematicamente } \\
\text { ensaios clínicos randomizados } \\
\text { sobre a efetividade das inter- } \\
\text { vençóes sobre os resultados do } \\
\text { exercício para pessoas com DP. }\end{array}$ & $\begin{array}{l}\text { Foi evidenciado os benefícios do } \\
\text { exercício para portadores da DP. }\end{array}$ \\
\hline $\begin{array}{l}\text { Fisioterapia respiratória } \\
\text { na doença de Parkinson } \\
\text { idiopática: relato de caso. }\end{array}$ & Alves LA. $^{22}$ & 2005 & $\begin{array}{l}\text { Relatar a evolução do caso } \\
\text { de um paciente parkinsonia- } \\
\text { no no estágio IV da Escala } \\
\text { de Hoehn e Yahr em que a } \\
\text { intervençáo fisioterapêutica foi } \\
\text { especificamente direcionada às } \\
\text { disfunçôes respiratórias. }\end{array}$ & $\begin{array}{l}\text { O pcte apresentou melhora } \\
\text { importante dos sintomas res- } \\
\text { piratórios e da prova de funçáo } \\
\text { pulmonar após tratamento } \\
\text { fisioterapêutico direcionado às } \\
\text { disfunçôes respiratórias. }\end{array}$ \\
\hline $\begin{array}{l}\text { Effects of treadmill training } \\
\text { on walking economey in } \\
\text { Parkinson`s disease: a pilot } \\
\text { study. }\end{array}$ & Pelosin E. ${ }^{23}$ & 2009 & $\begin{array}{l}\text { Avaliou os efeitos do treina- } \\
\text { mento na esteira e cicloergô- } \\
\text { metro. }\end{array}$ & $\begin{array}{l}\text { O treinamento melhorou signi- } \\
\text { ficativamente o desempenho da } \\
\text { caminhada. }\end{array}$ \\
\hline $\begin{array}{l}\text { Exercise performance in } \\
\text { those having Parkinson`s } \\
\text { disease and healthy normal. }\end{array}$ & Stanley RK. ${ }^{24}$ & 1999 & $\begin{array}{c}\text { Avaliar e comparar a função } \\
\text { cardiopulmonar de indivíduos } \\
\text { com DP com a de indivíduos } \\
\text { saudáveis. }\end{array}$ & $\begin{array}{l}\text { Os indivíduos com DP foram } \\
\text { menos eficientes durante o } \\
\text { exercício. }\end{array}$ \\
\hline
\end{tabular}

\section{DISCUSSÃO}

A DP afeta e prejudica as estruturas e as funçôes do corpo, causando limitaçóes nas atividades de vida diária, bem como o caminhar em casa e na comunidade, trocas de posturas, como para a postura ortostática, girar-se sobre o corpo, etc. Atividades funcionais que requerem desempenho nas habilidades motoras tornam-se comprometidas, mesmo que as habilidades para realizar movimentos simples permaneçam. Isto ocorre porque movimentos simples são controlados pelas regióes frontal, cerebelar e do tronco encefálico, mais do que pelos gânglios da base, e estas regiōes não são afetadas nos estágios iniciais da $\mathrm{DP}^{7}$.

Secundário à idade, imobilidade e desuso, a locomoção também pode ser afetada por prejuízos musculoesqueléticos, tais como a fraqueza muscular, diminuição da amplitude de movimento (ADM), tanto quanto a redução da capacidade aeróbica. Ao mesmo tempo, estas alteraçóes e limitaçóes restringem a capacidade da pessoa de participar em atividades sociais, como no trabalho, na educação, no lazer e até mesmo na vida pessoal ${ }^{7}$.

Como essa doença é progressiva, as intervençóes de exercícios não devem ser em curto prazo, mas se tornar parte do estilo de vida diário. Muitos clínicos e pesquisadores acreditam que a fisioterapia deve começar táo cedo quanto o estabelecimento do diagnóstico, para prevenir a atrofia muscular, a fraqueza e a capacidade de exercício reduzida $^{11,12}$.

O sedentarismo é visto atualmente como um problema mundial de saúde. Entre as razóes que levam à inatividade, está o desconhecimento sobre como se exercitar, as finalidades de cada exercício, limitaçôes de alguns gru- 
pos populacionais e percepçóes distorcidas em relação aos benefícios do movimento ${ }^{13}$.

Exercícios com treinamento repetitivos, ao serem realizados na fase inicial da doença, permitem um controle motor mais próximo do fisiológico e adequado quando houver maior deterioração da atuação dos gânglios da base, na evoluçấo natural da doença ${ }^{7}$.

Pelo menos um grande estudo epidemiológico demonstrou que pacientes fisicamente ativos tiveram uma menor taxa de mortalidade, melhor qualidade de vida e aumento das funçôes para as atividades de vida diária quando comparados com indivíduos com DP com menor atividade ${ }^{14}$.

Entre os vários benefícios promovidos pelo treinamento, o aumento do tônus e força dos músculos envolvidos na marcha e também o equilíbrio promoveram ao indivíduo com DP melhora em suas passadas, ficando estas mais alargadas e com utilização dos membros superiores. Este estudo demonstrou melhora no alinhamento biomecânico da sua postura, diminuição do tempo de execução de suas tarefas, aumentou a capacidade respiratória e a força dos músculos pulmonares, consequentemente, houve melhora no desempenho das funções pulmonares $^{15}$.

Um parâmetro prático da Academia Americana de Neurologia, de 2006, concluiu que várias modalidades terapêuticas físicas são provavelmente efetivas em melhorar o resultado funcional para pacientes com DP. Essas modalidades incluem: reabilitação multidisciplinar com componentes de fisioterapia e terapia ocupacional, treino na esteira com suspensão parcial de peso, treino de equilíbrio e de resistência de alta intensidade, exercícios sinalizados com audição e visão e feedback tátil e musicoterapia $^{16,17}$.

No momento, já existem profissionais que visam o acompanhamento multidisciplinar, contando com neurologistas experientes, fisioterapeutas doutores na área de DP e educadores físicos, indicando abordagens de tratamento domiciliares com exercícios físicos baseados em movimentos de diversas modalidades, tais como tai chi, boxe, canoagem e pilates. Este programa contempla a evolução da doença, com diferentes intensidades de exercícios, os quais são executados em um período de 60 minutos ${ }^{18}$.
Os pacientes com DP também possuem uma rigidez muscular global, acarretando alteraçóes da articulação dos fonemas e inteligibilidade de fala. Estas não podem ser evitadas, mas atenuadas com o encaminhamento precoce ao fonoaudiólogo, possibilitando ao paciente uma maior qualidade de vida com condiçóes mais satisfatórias de comunicação ${ }^{19}$.

Algumas revisóes sistemáticas e artigos conceituais mostraram que a fisioterapia tem como objetivo ensinar as pessoas com DP como minimizar os efeitos debilitantes músculo-esquelético e deficiências sensoriais, a fim de proporcionar uma melhor qualidade de vida e inclusão social ${ }^{7}$.

Em uma revisão realizada em 2001, mostra-se evidente que a fisioterapia contribuiu na reabilitação dos indivíduos com DP, diminuindo os efeitos debilitantes do comprometimento sensório-motor de maneira a melhorar a participação em atividades sociais e na qualidade de vida ${ }^{20}$

Em uma meta-análise comparando os achados em 14 estudos, também concluiu pela significância estatística da realização de exercícios físicos sobre a melhora na qualidade de vida, capacidade funcional, melhora na força muscular, no equilíbrio e na marcha. Da mesma maneira, não foi possível identificar melhoras no número de quedas e na depressão. Não foi possível quantificar o tipo, frequência e intensidade dos exercícios que auxiliam nos diferentes estágios da doença ${ }^{21}$.

Em um relato de caso o paciente com diagnóstico de DP, realizou durante cinco meses fisioterapia respiratória que consistia em exercícios respiratórios, mobilizaçóes e alongamentos. Foi realizado provas de função pulmonar (espirometria e manovacuometria) antes e após o período de tratamento e verificaram melhora da função respiratória com aumento nos valores de capacidade vital forçada, volume expiratório forçado no $1^{\mathrm{o}}$ segundo, pico de fluxo expiratório, pressão inspiratória e expiratória máxima e ventilação voluntária máxima ${ }^{22}$.

Outro aspecto importante a ser considerado é a função cardiorrespiratória. A atividade física, em particular, exercícios aeróbicos são de grande valia para pacientes com DP. Autores relatam que indivíduos com DP gastam aproximadamente $20 \%$ mais energia quando comparados a sujeitos sem o diagnóstico de DP, durante os 
exercícios na esteira e com ciclo ergômetro. Ao mesmo tempo em que descrevem uma melhora na economia de energia quando submetidos a treinamento de exercícios resistidos, em seu estudo com dez pacientes submetidos a treinamento em esteira durante quatro semanas, demonstrou uma melhora significativa na economia de energia e no pico de VO2 máximo ${ }^{23}$.

As anormalidades cardiovasculares, que são frequentemente vistas em pacientes com DP, incluem hipotensão ortostática, arritmia cardíaca, e menos frequentemente hipertensão. Em relação à arritmia cardíaca, os idosos, que são mais frequentemente afetados pela DP também têm a maior probabilidade de doença cardiovascular. Portanto, a diferenciação entre sintomas relacionados à idade e aqueles que podem ser associados à DP não são facilmente identificados. Arritmia cardíaca em indivíduos tendo DP também tem sido associada à quase todas as medicaçôes antiparkinsonianas. Embora essas medicaçôes possam causar arritmia, até o momento os estudos não obtiveram conclusôes definitivas sobre medicaçóes antiparkinsonianas causando toxidade cardíaca ${ }^{24,25}$.

Outro problema cardíaco associado com a DP é o comprometimento dos reflexos cardiovasculares, os quais podem causar respostas cardíacas anormais. Uma das respostas anormais naqueles com comprometimento severo dos reflexos cardiovasculares é uma frequência cardíaca fixa. Em casos individuais, onde há uma frequência cardíaca fixa, a taquicardia que ocorre em resposta a certos estímulos tais como o exercício, não ocorrerá. Portanto é de grande importância identificar aqueles indivíduos de maneira a monitorar restriçôes cardíacas ${ }^{10}$.

Diversas alterações fisiológicas em indivíduos com DP influenciam o sistema respiratório diretamente ou indiretamente. A rigidez muscular progressiva, que frequentemente acontece, não somente prejudica a musculatura das extremidades, mas também a musculatura esquelética axial. Consequentemente a rigidez da musculatura vertebral e do tórax, incluindo a caixa torácica e os músculos respiratórios, pode ter um efeito direto ou indireto na respiração normal. Outro adicional é envolvimento dos músculos faciais e cervicais, tanto quanto da região da garganta e do esôfago, podendo também afetar a respiração normal ${ }^{10,25}$.

\section{CONCLUSÃO}

A fisioterapia, orientando a prática de atividade física é de extrema importância para manter, melhorar e prolongar a qualidade de vida do indivíduo. Embora a limitação no tamanho das amostras não garanta de forma conclusiva que a aplicaçáo de qualquer um desses exercícios em outros pacientes com DP terá o mesmo resultado, havendo pouca evidência científica encontrada na literatura sobre o condicionamento físico em indivíduos com DP. Sugere-se que estudos com amostras maiores sejam realizados, os quais possam ou não corroborar com os achados na referida revisão.

\section{REFERÊNCIAS}

1.Meneses MS, Teive HAG. Doença de Parkinson: aspectos clínicos e cirúrgicos. Rio de Janeiro: Guanabara Koogan, 1996, 189p.

2.Perkin DG. An Atlas of Parkinson's Disease and Related Disorders. Londres: Parthenon Publishing Group, 1998, 96p.

3.Jankovic J. Parkinson's disease: clinical features and diagnosis. Journal of Neurology, Neurosurgery \& Psychiatry 2007;79:368-76.

http://dx.doi.org/10.1136/jnnp.2007.131045

4.Sant CR, Oliveira SG, Rosa EL, Sandri J, Durante M, Posser SR. Abordagem fisioterapêtica na Doença de Parkinson. RBCEH 2008;5:80-9.

5.Chiappetta ALML. Doenças neuromusculares, Parkinson e Alzheimer. São José dos Campos: Pulso, 2003, 73p.

6.Bahram Y, Vahid T, Fathollahzadeh KA, Ali M. Exercise therapy, quality of life, and activities of daily living in patients with Parkinson disease: a small scale quasi-randomised trial. Trials Journal 2009:10:67.

http://dx.doi.org/10.1186/1745-6215-10-67

7.Morris ME. Locomotor Training in People With Parkinson Disease. Journal of the American Physical Therapy Association 2006;86:1426-35.

8.Goulart F, Pereira LX. Uso de Escalas para Avaliação da Doença de Parkinson em Fisioterapia. Fisioterapia e Pesquisa 2005;11:49-56.

9.Canning GC, Sherrington C, Lord SR, Fung VSC, Close JCT, Latt MD, et al. Exercise therapy for prevention of falls in people with Parkinson's disease: A protocol for a randomised controlled trial and economic evaluation. BMC Neurology 2009;9:4.

http://dx.doi.org/10.1186/1471-2377-9-4

10.Reuter I, Engelhardt M. Exercise training and Parkinson`s disease. The physician and Sportsmedicine 2002;30:1-8.

http://dx.doi.org/10.3810/psm.2002.03.200

11.Morris ME. Movement disorders in people with Parkinson disease: a model for physical therapy. Journal of the American Physical Therapy Association 2000;80:579-97.

12.Reuter I, Engelhardt M, Stecker K, Baras H. Therapeutic value of exercise training in Parkinson`s disease. Medicine \& Science in Sports and Exercise 1999;31:1544-9.

http://dx.doi.org/10.1097/00005768-199911000-00008

13.Domingues MR, Araújo CLP, Gigante DP. Conhecimento e percepção sobre exercício físico em uma população adulta urbana do sul do Brasil. Caderno 
Saúde Pública 2004;20:204-15.

http://dx.doi.org/10.1590/S0102-311X2004000100037

14.Johnson AM, Almeida QJ. The impacto of exercise rehabilitation and physical activity on the management of Parkinson`s disease. Geriatrics \& Aging 2007;10:318-21.

15.Braga A, Xavier ALIL, Machado RPO. Benefícios do Treinamento Resistido na reabilitação da Marcha e Equilíbrio nos Portadores de Doença de Parkinson. Revista Digital Vida \& Saúde, Juiz de Fora. 2003;2:2.

16.Tarsy D, Hurtig HI, Dashe JF. Nonpharmacologic management of Parkinson disease. Uptodate: last literature review 2008;1-5.

17.Wade DT. Multidiciplinary rehabilitation for people with Parkinson`s disease: a randomized controlled study. Journal of Neurology, Neurosurgery \& Psychiatry with Pratical Neurology 2002;74:158-62.

http://dx.doi.org/10.1136/jnnp.74.2.158

18.King LA, Fay B H. Delaying Mobility Disability in People With Parkinson Disease Using a Sensorimotor Agility Exercise Program. Journal of the American Physical Therapy Association 2009; 89:384-93.

19.Barros ALS, Silveira EGC, Souza RCM, Freitas LC. Uma Análise do Comprometimento da Fala em Portadores da Doença de Parkinson. Rev Neuro- cienc 2004;12:123-9.

20.Deane K, Jones D, Ellis-Hill C. Physiotherapy for patients with Parkinson's disease. Cochrane Database Systematic Reviews 2001;3:CD002815.

21.Goodwin VA, Richards SH, Taylor RS, Taylor AH, Campbell JL. The effectiveness of exercise interventions for people with Parkinson disease: a systematic review and meta-analysis. Mov Disord 2008;23:631-40.

http://dx.doi.org/10.1002/mds.21922

22.Alves LA, Coelho AC, Brunetto AF. Fisioterapia Respiratória na Doença de Parkinson Idiopática: relato de caso. Fisioterapia e Pesquisa 2005;12:46-9. 23.Pelosin E, Faelli E, Lofrano F, Avanzino L, Marinelli L, Bove M. Effects of treadmill training on walking economy in Parkinson'sdisease: a pilot study. Neurol Sci 2009;30:499-04.

http://dx.doi.org/10.1007/s10072-009-0141-8

24.Stanley RK, Protas EJ, Jankovic J. Exercise performance in those having Parkinson`s disease and healthy normals. Med Sci Sports Exerc 1999;31:761-6. http://dx.doi.org/10.1097/00005768-199906000-00001

25.Deturk WE, Cahalin LP. Fisioterapia cardiorrespiratória: cuidados cardiorrespiratórios em pacientes com déficit neurológico- uma abordagem baseada em evidências. Porto Alegre: Artmed, 2004, p.427-30. 